\title{
Enhanced Performance of InAsP Nanowires with Ultra-thin Passivation Layer
}

\author{
$\underline{\text { Stephanie O. Adeyemo }}^{1}$, Srabani Kar ${ }^{1}$, Yunyan Zhang ${ }^{2}$, Huiyun Liu $^{2}$ and Hannah J. Joyce ${ }^{1, *}$ \\ ${ }^{1}$ Electrical Engineering Division, Department of Engineering, University of Cambridge, $9 \mathrm{~J}$ J Thomson Ave, Cambridge, CB3 0FA UK \\ ${ }^{2}$ Department of Electronic and Electrical Engineering, University College London, London, WC1E 7JE UK \\ *Corresponding Author: hjj28@cam.ac.uk
}

\begin{abstract}
Surface passivation with a higher band gap shell has been shown to successfully reduce the density of surface states at the surface of nanowires. The effect of ultra-thin InP passivation layers of thicknesses $\sim 3-5 \mathrm{~nm}$ coated on InAsP nanowires is investigated and compared to bare InAsP nanowires. The ultrathin passivation exhibited an improvement in carrier lifetime and mobility by approximately a factor of 3 . Surface recombination velocity was decreased by at least a factor of 3 .
\end{abstract}

\section{INTRODUCTION}

$\mathrm{S}$ EMICONDUCTOR nanowires are favourable materials for nanoscale electronic and optoelectronic applications because of their excellent and unique properties. However, due to the large surface area-to-volume ratio intrinsic to nanowires, the electronic properties of unpassivated nanowires are strongly sensitive to surface states acting as a source of nonradiative carrier trapping sites within the band gap resulting in rapid decay of photoexcited carriers ${ }^{1,2}$. These surface states have normally been effectively suppressed by passivating the nanowires with a higher band gap material shell while maintaining optimal lattice matching between the core and shell. Usually, the passivation layer is about tens of nanometres thick, however, this makes it difficult to implement on a highly strained material and can also be detrimental for implementation in some devices. Thus, it is critical to achieve effective passivation using minimally thick films.

Here, we have carried out transient terahertz conductivity measurements on unpassivated and passivated InAsP nanowires to assess and demonstrate the effect of an ultra-thin InP passivation layer on charge carrier lifetime, mobility, surface recombination velocity and photoconductivity. We have employed optical pump-terahertz probe (OPTP) spectroscopy, a non-contact room temperature technique ${ }^{3}$, to investigate and compare the carrier dynamics of InAsP nanowires of core diameter $\sim 45 \mathrm{~nm}$ with that of InAsP nanowires passivated with ultra-thin InP shells of thicknesses $\sim 3-5 \mathrm{~nm}$. We employed a photoexcitation energy of $\sim 0.59 \mathrm{eV}$, just above the InAsP band gap. All measurements were carried out at room temperature and under dry atmosphere purged with nitrogen.

\section{RESULTS \& DISCUSSION}

The scanning electron microscopy (SEM) images of the bare and passivated nanowires are shown in figure $1 \mathrm{a}$ and $1 \mathrm{~b}$ respectively. The photoconductivity decays $\Delta \sigma(\mathrm{t})$ obtained from transient terahertz conductivity measurements on the InAsP and InAsP/InP nanowires shown in figure 1c were fitted with single exponential functions at early times to assess surface recombination when the surface traps are originally unoccupied. The recombination in bare InAsP nanowires is clearly dominated by Shockley-Read-Hall (SRH) mechanism and the charge carriers decay rapidly on a timescale with lifetime $\tau$ of $\sim 120 \mathrm{ps}$. The passivated nanowires show considerably slower recombination due to the reduction of the surface states density by the highly effective ultra-thin InP shell. Charge carriers of the passivated nanowires decay slowly on a nanosecond timescale featuring a longer carrier lifetime $\tau$ of $\sim 340 \mathrm{ps}$, therefore revealing an increase in the carrier lifetime by approximately a factor of 3 through the surface passivation technique. Additionally, the InP passivation enormously lowers the surface recombination velocity $S$ by a factor of 3 compared to the bare InAsP nanowires leading to an enhancement of radiative recombination in the InAsP nanowires.
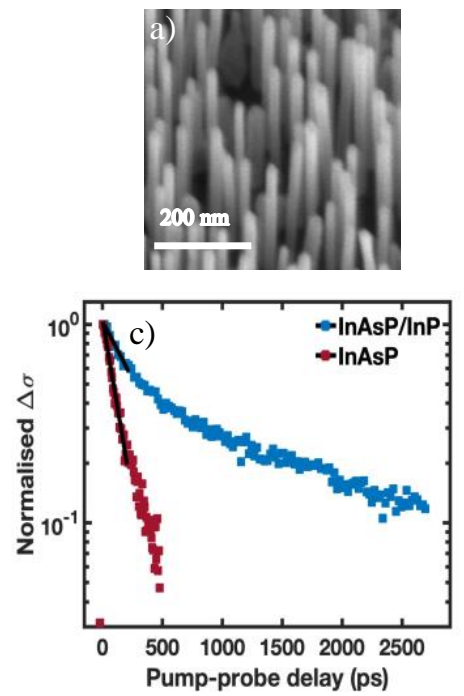
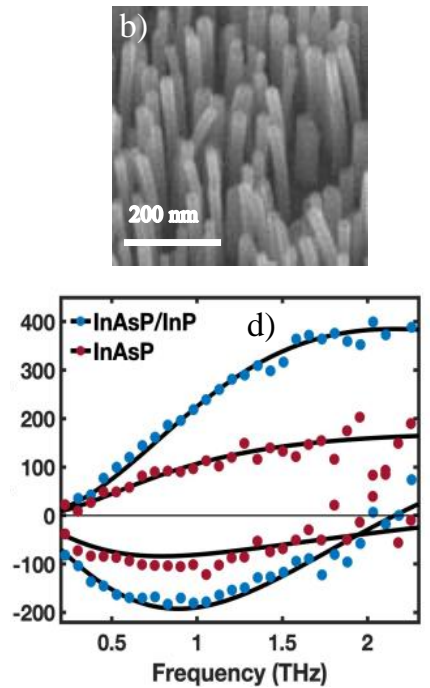

Figure 1: Scanning electron microscopy (SEM) images of (a) bare InAsP nanowires of diameters $\sim 45 \mathrm{~nm}$ and (b) Passivated InAsP nanowires of InP shell thicknesses $\sim 3-5 \mathrm{~nm}$. The images were taken at a tilt of $30^{\circ}$. Scale bar is $200 \mathrm{~nm}$ for both samples. (c) Normalised photoconductivity decays of both nanowire samples on a semi-logarithmic scale. Straight black lines are monoexponential fits. (d) Photoconductivity spectra $\Delta \sigma(\omega)$ of both nanowire samples showing a strong Lorentzian response. Real and imaginary parts of the measured $\Delta \sigma(\omega)$ are plotted.

The photoconductivity spectra $\Delta \sigma(\omega)$ of both nanowire samples at $20 \mathrm{ps}$ after photoexcitation is presented in figure $1 \mathrm{~d}$ The $\Delta \sigma(\omega)$ spectra exhibited a strong Lorentzian response which is typical of plasmon modes of semiconductor nanostructures ${ }^{3,4}$. The Lorentzian function is described by

$$
\sigma(\omega)=\frac{N e^{2}}{m^{*}} \frac{\mathbf{i} \omega}{\omega^{2}-\omega_{0}^{2}+\mathbf{i} \omega \gamma}
$$

where $N$ is the charge carrier density, $e$ is the electronic charge, $m^{*}$ is the effective mass, $\omega_{0}$ is the resonant frequency and $\gamma$ is the scattering rate. The spectra were fitted with the Lorentzian 
function to extract the scattering rate $\gamma$. The scattering rate $\gamma$ was therefore used to calculate the electron mobilities $\mu$ of both nanowires by $\mu=e / m^{*} \gamma$, where $e$ is the electronic charge and $m^{*}$ is the effective mass. Using this relation, the extracted carrier mobilities are $\sim 2400 \mathrm{~cm}^{2} \mathrm{~V}^{-1} \mathrm{~s}^{-1}$ for the passivated nanowires and $\sim 800 \mathrm{~cm}^{2} \mathrm{~V}^{-1} \mathrm{~s}^{-1}$ for the bare InAsP nanowires. Therefore, the surface passivation technique has improved the carrier mobility by a factor of 3 .

\section{ACKNOWLEDGEMENT}

The authors would like to acknowledge financial support from the European Research Council (ERC Starting Grant ACrossWire) and the Engineering and Physical Sciences Research Council (UK). We gratefully acknowledge the Warwick Centre for Ultrafast Spectroscopy for access to facilities.

\section{REFERENCES}

[1]. H.J. Joyce, C.J. Docherty, Q. Gao, H.H. Tan, C. Jagadish, J. Lloyd-Hughes, L.M. Herz and M.B. Johnston, "Electronic properties of GaAs, InAs and InP nanowires studied by terahertz spectroscopy," Nanotechnology, vol. 24, 214006, 2013.

[2]. J.A. Alexander-Webber, C.K. Groschner, A.A. Sagade, G. Tainter, M.F Gonzalez-Zalba, R. Di Petro, J. Wong-Leung, H. H. Tan, C. Jagadish, S. Hofmann and H.J. Joyce, "Engineering the photoresponse of InAs Nanowires," ACS Applied Materials \& Interfaces, 9 (50), 43993 - 44000, 2017.

[3]. H.J. Joyce, J.L. Boland, C.S. Davies, S.A. Baig and M.B. Johnston, “A review of the electrical properties of semiconductor nanowires: insights gained from terahertz conductivity spectroscopy," Semicon. Sci. and Technology, vol. 34, 103003, 2016.

[4]. H-K Nienhuys and V. Sunström, "Influence of plasmons on terahertz conductivity measurements," Appl. Phys. Lett., vol. 87, 012101, 2007. 